Statistics:

Estimated number of words: 3500
Number of Figures: 5

Number of Tables: 0

\title{
Interaction between charged lipid vesicles and point- or rod-like trivalent ions
}

\author{
Alberto Martin-Molina \\ Departamento de Fisica Aplicada and Instituto Carlos I de Fisica Teorica y Computacional, \\ Facultad de Ciencias, Universidad de Granada, 18071 Granada, Spain \\ Leo Lue \\ Department of Chemical and Process Engineering, \\ University of Strathclyde, Glasgow G1 $1 X J$, UK \\ Manuel Quesada-Perez \\ Departamento de Fisica, Escuela Politecnica Superior de Linares, \\ Universidad de Jaen, 23700, Linares, Jaen, Spain \\ Klemen Bohinc* \\ Faculty of Health Sciences, University of Ljubljana, Zdravstvena 5, SI-1000 Ljubljana, Slovenia
}

(Dated: January 25, 2019)

\begin{abstract}
This work examines the influence of the charge distribution of trivalent cations when they interact with soft anionic particles, using a combination of experimental measurements and theoretical modelling. In particular, we perform electrophoresis measurements to calculate the zeta-potential of anionic liposomes in the presence of spermidine and lanthanum cations. We work in a range of electrolyte concentration where we expect a reversal in the electrophoretic mobility. However, unlike the case of trivalent lanthanum cations, spermidine does not induce mobility reversal of liposomes. As a result, charge distribution within the counterion appears as a key factor in the appearance of such phenomenon. This conclusion is supported by a theory that accounts for intra-ionic correlations. This theory has been successfully used to describe the colloidal electric double layer, previously. In fact, the theory allows us to model spermidine as rod-like ions and lanthanum cations as point-like ions in order to test the importance of the ionic geometry in the interactions with soft particles such as lipid vesicles.
\end{abstract}

\section{INTRODUCTION}

The interaction between multivalent cations and lipids plays an essential role in the structure and function of biological membranes. For instance, it has been shown that multivalent cations can mediate membrane fusion [1-4] and fractal aggregation $[5,6]$ by interacting with negatively charged phospholipids. It has been also demonstrated that multivalent counterions can induce charge reversal $(\mathrm{CR})$ on lipids membranes [7-15]. This phenomenon (also known as overcharging or charge inversion) occurs when counterions are attracted to a charged interface in excess of its own bare charge. The driving force for this counter-intuitive phenomenon has been extensively discussed, but there is strong evidence supporting the view that ion-ion correlations play a major role in the case of hard particles [16-21].

In contrast, for soft particles, such as lipid vesicles (liposomes), a new mechanism of CR that operates only for ions capable of penetrating into soft interfaces has been proposed $[14,15]$. The $\mathrm{CR}$ in anionic liposomes at low concentrations of multivalent spherical cations has been

\footnotetext{
*klemen.bohinc@zf.uni-lj.si
}

demonstrated by electrophoresis measurements. Furthermore, all-atomic molecular dynamics (MD) simulation results indicated that the underlying mechanism of $\mathrm{CR}$ for this system is the preferential solvation of counterions by amphiphilic molecules and hydration water. Therefore, solvent mediated effects can be strong enough to govern the electrostatics of soft interfaces [14]. These solvent effects are not considered in most of the ion-ion correlation theories based on the primitive model of electrolyte, which have been widely used to explain $\mathrm{CR}$ in model colloids [22-28].

The primitive model treats ions as charged hard spheres immersed in a dielectric continuum and, consequently, is not appropriate for the case of non-spherical multivalent ions. For these systems, additional methods are required to account for the internal structure of the ions [29? -31]. An example of non-spherical multivalent ion is the case of polyamine spermidine, which is very relevant in biophysics since it can induce DNA condensation [32]. Multivalent ion-induced DNA condensation was first observed with the naturally occurring polyamine spermidine which, together with other polyamines, is involved in several cellular processes including DNA compaction in vivo [33, 34]. Accordingly, there has been much interest in the interactions of spermidine with nano- and micro-particles, in the last decades 
(see [30, 31, 35-37] and references cited therein). For instance, silica particle interactions in the presence of spermidine were recently investigated both from experimental and theoretical points of view [31]. However, as previously commented, the interaction between multivalent ions with hard particles can be very different to that found for soft particles.

To this end, electrophoresis experiments of anionic liposomes in the presence of lanthanum and spermidine cations have been performed. More specifically, the variation of the electrophoretic mobility as a function of the electrolyte concentration is measured. In the past, electrophoresis experiments have been used to explore situations at which mobility reversal is induced. Ottewill and Shaw carried out an extensive, pioneering study of colloidal dispersions containing trivalent and tetravalent counterions [38]. More recently, mobility reversals have also been reported for other colloids in 3:1 electrolytes $[14,17,22,23]$. Apart from electrophoresis measurements, experimental data are fitted by using a single point representation for the case of lanthanum whereas a rod-like ion model is applied for the spermidine measurements.

Mean-field theory does not include charge-charge correlations and does not predict proper distributions of multivalent counterions close to the charged surfaces [39]. The description can be improved by accounting for interion correlations between (multivalent) counterions. The correlations between point-like multivalent ions has attracted considerable interest in the past [40, 41]. Recently a field theoretic approach was proposed that can accurately describe the properties of charged systems from the weak to the intermediate and the strong coupling regimes $[42,43]$.

Mobile ions can have internal structure with a spatially distributed charge. Examples include polyamines like diamin, spermidine, and spermine. The theory was generalized to systems with polydisperse rod lengths and arbitrary charge distributions along the rods [30, 44-46]. It was demonstrated that intra-ionic correlations induced by the fixed distance within a particular rod-like ion can change repulsive into attractive interactions between like charged surfaces. The minimum of the free energy appears when the counterions are oriented perpendicularly in order to connect the like-charged surfaces. Monte Carlo simulations confirmed the theoretical predictions [47-49]. The analysis of the system was later extended to the intermediate and strong coupling regimes, where the interionic correlations alone can lead to an attraction between the surfaces $[30,50,51]$.

In this work, we analyze electrophoretic mobility measurements of anionic soft particles in the presence of trivalent salts with different ionic geometry (see Fig. 1), using a field theory that accounts for intra-ionic and inter-ionic correlations. Accordingly, the goal of this work is twofold. On one hand, the interaction of spherical trivalent cations and anionic soft particles is studied. On the other hand, results with rod-like trivalent cations are provided and compared to previous ones. To this end, electrophoresis experiments of anionic liposomes in the presence of lanthanum and spermidine cations have been performed. More specifically, the variation of the electrophoretic mobility as a function of the electrolyte concentration is measured.

The remainder of this paper is organized as follows. In the next section, we provide the details of the electrophoretic mobility measurements of liposomes performed in this work. Then in Sec. III, we describe the splitting theory and its application to describe the surface charge potential of a uniformly charged plate that is immersed in a solution of point charges and rod-like counterions. This theory is used to predict the zeta-potential of the liposome (macroion). The results from the electrophoretic mobility experiments are presented in Sec. IV and compared with the predictions of the splitting theory. Finally, the main findings of this work are summarized in Sec. V.

\section{MATERIAL AND METHODS}

The lipids used in this work are anionic PS lipids (3sn-Phosphatidyl-L-serine from bovine brain) and zwitterionic PC lipids (egg phosphatidylcholine with purities $>99 \%$ ) purchased from Sigma-Aldrich. Spermidine electrolyte $\left(\mathrm{C}_{7} \mathrm{H}_{19} \mathrm{~N}_{3}\right)$ (with a purity of 99\%) was acquired also from Sigma-Aldrich. $\mathrm{La}\left(\mathrm{NO}_{3}\right)_{3}$ salt (with purities $>98.6 \%$ ) and reagent grade chloroform and methanol were acquired from Scharlau Chemie S.A. (Spain). Ultrapure water, cleaned using a MilliQ (Millipore, USA) water purification system $(0.054 \mu \mathrm{S})$, was used for the preparation of samples. All solutions were prepared at $298 \mathrm{~K}$.

\section{A. Characterization of the liposomes}

Dry films of mixed lipids were prepared by dissolving PS and PC lipids in a molar ratio of 1:1, in $10 \mathrm{ml}$ of chloroform/methanol (3:1 by volume). The solvent was evaporated under vacuum by rotary evaporation for at least $2 \mathrm{~h}$ and then passed through a steady stream of nitrogen gas $\left(\mathrm{N}_{2}\right)$. The dry film was hydrated with deionized water (MilliQ, Millipore, USA) to give the desired lipid concentration. Multilamellar liposomes were formed by continuous mixing with a vortex mixer and sonication. Finally, small unilamellar vesicles were formed by using the extrusion technique, which consists in 5 passes through a $800 \mathrm{~nm}$ pore size polycarbonate membrane followed by 5 passes through a $200 \mathrm{~nm}$ pore size polycarbonate membrane. The extrusion was carried out using a Thermobarrel Lipex Extruder (Northern Lipids Inc., Canada), with a capacity of $10 \mathrm{ml}$ and that uses a flow of $\mathrm{N}_{2}$ under pressure to force the solution through the membrane. The final liposome solution was stored in the fridge at $277-280 \mathrm{~K}$. The size 
FIG. 1. Schematic illustration of negatively charged lipid vesicle embedded in a solution of spermidine ions and monovalent co-ions (left). Charged lipid vesicles in a solution of lanthanum ions and monovalent coions (right).

distribution of the sample was tested before each experiment by photon correlation spectroscopy (PCS), yielding a mean particle size of $144 \pm 14 \mathrm{~nm}$ with a standard deviation of $0.28 \pm 0.15 \mathrm{~nm}$.

\section{B. Electrokinetic experiments}

A ZetaPALS (Brookhaven, USA) instrument, which uses phase analysis light scattering (PALS), was used to measure electrophoretic mobilities $\left(\mu_{e}\right)$. This technique is fully described in Ref. [15]. Our electrophoretic mobility measurements were performed at $298 \mathrm{~K}$. The $\mathbf{p H}$ was measured to be $\mathbf{5 . 5}$ both before and after the measurements to check that it did not change, following the procedure given in Ref. 38. As in a previous study [52], the zeta-potential was obtained from the electrophoretic mobility by using the Henry equation. The liposome concentration was $5 \times 10^{-4} \mathrm{M}$, and the electrolyte concentration varied from $10^{-6}$ to $10^{-1} \mathrm{M}$. The reproducibility of the experiments was tested by repeating each measurement at least three times with independent samples.

\section{THEORY}

In order to examine the interaction of the ions with the lipid vesicle, we consider a planar surface with a uniform negative surface charge density $\Sigma$. The surface is embedded in an aqueous solution containing either pointor rod-like counterions and small monovalent co-ions. These mobile charges are restricted to lie between the surfaces. A schematic representation of the system is given in Fig. 1. The coordinate system is defined such that the $x$-axis and $y$-axis are parallel to the surfaces, and the $z$-axis is perpendicular to the surfaces. The origin is located on the left surface.

The total charge density $\mathcal{Q}$ in the system is given by

$$
\mathcal{Q}(\mathbf{r})=\varrho(\mathbf{r})+\Sigma \delta(z)
$$

where the first term on the right side of the equation corresponds to the charge density of the mobile counterions and co-ions, and the second term corresponds to the fixed surface charge on the plate. The charge density $\varrho(\mathbf{r})$ of the mobile ions is given by

$$
\begin{aligned}
\varrho(\mathbf{r})= & q \sum_{k}\left[\delta^{d}\left(\mathbf{r}-\mathbf{R}_{c, k}+\hat{\mathbf{n}}_{c, k} l / 2\right)+\delta^{d}\left(\mathbf{r}-\mathbf{R}_{c, k}\right)\right. \\
& \left.+\delta^{d}\left(\mathbf{r}-\mathbf{R}_{c, k}-\hat{\mathbf{n}}_{c, k} l / 2\right)\right] \\
& -\sum_{k} q \delta^{d}\left(\mathbf{r}-\mathbf{R}_{s, k}\right),
\end{aligned}
$$

where $\mathbf{R}_{c, k}$ is the location of the center of the $k$ th rod-like counterion, $\hat{n}_{c, k}$ is its orientation, $\mathbf{R}_{s, k}$ is the position of the $k$ th monovalent co-ion, and $q$ is the magnitude of the charge. The rod-like counterions are trivalent and have an overall length $l$; each consists of three point charges: one located at its center, and one on either end. The case of a point-like counterion corresponds to $l=0$.

In our model, the only interactions in the system are electrostatic interactions

$$
\begin{aligned}
E^{\mathrm{elec}}= & \frac{1}{2} \int d \mathbf{r} d \mathbf{r}^{\prime} \mathcal{Q}(\mathbf{r}) G\left(\mathbf{r}, \mathbf{r}^{\prime}\right) \mathcal{Q}(\mathbf{r}) \\
& -\sum_{k=1} e_{c}^{\mathrm{se}}\left(\mathbf{R}_{c, k}, \hat{\mathbf{n}}_{c, k}\right)-\sum_{k=1} e_{s}^{\mathrm{se}}\left(\mathbf{R}_{s, k}\right)
\end{aligned}
$$

where $G\left(\mathbf{r}, \mathbf{r}^{\prime}\right)=\left|\mathbf{r}-\mathbf{r}^{\prime}\right|^{-1}$ is the Green's function of the Poisson equation, $e_{c}^{\mathrm{se}}(\mathbf{R}, \hat{\mathbf{n}})$ is the self energy of a counterion, and $e_{s}^{\mathrm{se}}(\mathbf{R})$ is the self energy of a co-ion.

In order develop an accurate approximation for the static properties of the system, we split the Green's function of the electrostatic interactions into shortwavelength $G_{s}$ and long-wavelength $G_{l}$ contributions $[42,50]: G\left(\mathbf{r}, \mathbf{r}^{\prime}\right)=G_{s}\left(\mathbf{r}, \mathbf{r}^{\prime}\right)+G_{l}\left(\mathbf{r}, \mathbf{r}^{\prime}\right)$, where $G_{l}=\mathcal{P} G$, and $G_{s}=(1-\mathcal{P}) G$, and $\mathcal{P}$ is an operator that filters out the short-wavelength fluctuations. For this operator, we have chosen $\mathcal{P}=\left[1-\sigma^{2} \nabla^{2}+\sigma^{4} \nabla^{4}\right]^{-1}$, where the parameter $\sigma$ is a length scale which distinguishes between short-wavelength and long-wavelength phenomena. With this choice, the short-ranged Green's function is

$$
\begin{aligned}
G_{s}\left(\mathbf{r}, \mathbf{r}^{\prime}\right)= & \frac{1}{\left|\mathbf{r}-\mathbf{r}^{\prime}\right|} e^{-\sqrt{3}\left|\mathbf{r}-\mathbf{r}^{\prime}\right| /(2 \sigma)} \\
& \times\left[\cos \frac{\left|\mathbf{r}-\mathbf{r}^{\prime}\right|}{2 \sigma}+\frac{1}{\sqrt{3}} \sin \frac{\left|\mathbf{r}-\mathbf{r}^{\prime}\right|}{2 \sigma}\right],
\end{aligned}
$$

and the long-ranged Green's function is

$$
\begin{aligned}
G_{l}\left(\mathbf{r}, \mathbf{r}^{\prime}\right)= & \frac{1}{\left|\mathbf{r}-\mathbf{r}^{\prime}\right|} \\
& -\frac{1}{\left|\mathbf{r}-\mathbf{r}^{\prime}\right|} e^{-\sqrt{3}\left|\mathbf{r}-\mathbf{r}^{\prime}\right| /(2 \sigma)} \\
& \times\left[\cos \frac{\left|\mathbf{r}-\mathbf{r}^{\prime}\right|}{2 \sigma}+\frac{1}{\sqrt{3}} \sin \frac{\left|\mathbf{r}-\mathbf{r}^{\prime}\right|}{2 \sigma}\right]
\end{aligned}
$$

With the division of the Green's function, the grand partition function of the system can be written as a functional integral over short-wavelength $\psi_{s}$ and longwavelength $\psi_{l}$ electrostatic interactions. The functional integration over the field $\psi_{s}$ is evaluated by a first-order cumulant expansion. The functional integration over the 
field $\psi_{l}$ is evaluated using the mean-field approximation $[42,53]$. The resulting approximation for the free energy $F$ of the system is

$$
\begin{aligned}
\frac{1}{A} F= & \frac{\beta}{A} \int d \mathbf{r} d \mathbf{r}^{\prime} \Sigma(\mathbf{r}) G_{s}\left(\mathbf{r}, \mathbf{r}^{\prime}\right) \Sigma\left(\mathbf{r}^{\prime}\right) \\
& +\int d \mathbf{R} d \hat{\mathbf{n}} \rho_{c}(\mathbf{R}, \hat{\mathbf{n}}) \\
& \times\left[\ln \rho_{c}(\mathbf{R}, \hat{\mathbf{n}}) \Lambda_{c}^{d}-1+\beta u_{c}(\mathbf{R}, \hat{\mathbf{n}})\right] \\
& +\int d \mathbf{R} \rho_{s}(\mathbf{R})\left[\ln \rho_{s}(\mathbf{R}) \Lambda_{s}^{d}-1+\beta u_{s}(\mathbf{R})\right] \\
& -\frac{1}{2} \int d \mathbf{r} d \mathbf{r}^{\prime} i \bar{\psi}_{l}(\mathbf{r}) G_{l}^{-1}\left(\mathbf{r}, \mathbf{r}^{\prime}\right) i \bar{\psi}_{l}\left(\mathbf{r}^{\prime}\right) \\
& +\int d \mathbf{r} \mathcal{Q}(\mathbf{r}) i \bar{\psi}_{l}(\mathbf{r})
\end{aligned}
$$

where $A$ is the area of the plates, $\Lambda_{c}$ is the thermal wavelength of the counterions, $\Lambda_{s}$ is the thermal wavelength of the co-ions, $\beta=\left(k_{B} T\right)^{-1}\left(k_{B}\right.$ is the Boltzmann constant and $T$ is the absolut temperature of the system $), \rho_{c}(\mathbf{R}, \hat{\mathbf{n}})$ is the counter density, $\rho_{s}(\mathbf{R})$ is the co-ion density, $\mathcal{Q}(\mathbf{r})$ is the charge density in the system

$$
\mathcal{Q}(\mathbf{r})=q \sum_{m=0, \pm 1} \rho_{c}(\mathbf{R}+m \hat{\mathbf{n}} l / 2, \hat{\mathbf{n}})-q \rho_{s}(\mathbf{r}),+\Sigma \delta(z)
$$

$u_{c}(\mathbf{R}, \hat{\mathbf{n}})$ is the one-body potential for the counterions

$$
\begin{aligned}
u_{c}(\mathbf{R}, \hat{\mathbf{n}})= & q \sum_{m=0, \pm 1} \int d \mathbf{r} G_{s}(\mathbf{R}+m \hat{\mathbf{n}} l / 2, \mathbf{r}) \Sigma(\mathbf{r}) \\
& -\frac{q^{2}}{2} \sum_{m, m^{\prime}=0, \pm 1} G_{l}\left(\mathbf{R}+m \hat{\mathbf{n}} l / 2, \mathbf{R}+m^{\prime} \hat{\mathbf{n}} l / 2\right),
\end{aligned}
$$

and $u_{s}(\mathbf{R})$ is the

$$
\begin{aligned}
u_{s}(\mathbf{R})= & -q \int d \mathbf{r} G_{s}(\mathbf{R}, \mathbf{r}) \Sigma(\mathbf{r}) \\
& -\frac{q^{2}}{2} G_{l}(\mathbf{R}, \mathbf{R})
\end{aligned}
$$

The value of $\sigma$ is determined by making the free energy stationary:

$$
\frac{\partial F}{\partial \sigma}=0
$$

The electrostatic potential $\phi(\mathbf{r})$ is given by the solution of the Poisson equation:

$$
-\frac{\varepsilon}{4 \pi} \nabla^{2} \phi(\mathbf{r})=\mathcal{Q}(\mathbf{r})
$$

where $\varepsilon$ is the dielectric constant of the solvent (i.e. water).
FIG. 2. Concentration profiles of spermidine/point-like ions (dashed lines) and monovalent coions (solid lines) at $c=$ $10^{-4} \mathrm{M}$ (red), $c=10^{-3} \mathrm{M}$ (black), and $c=10^{-2}$ (blue). The thick lines correspond to spermidines with associated coions profiles, whereas the thin lines correspond to point-like ions with associated coion profiles. The Bjerrum length is $l_{B}=$ $0.7 \mathrm{~nm}$.

\section{RESULTS AND DISCUSSION}

The vesicles are large compared to the spermidine molecules, and we can consider the behavior of spermidine molecules close to the charged planar surface. Assuming that all lipids form liposomes, we calculate that there are 35254 lipids per liposome, based on the charge measurements. Given that the average size of the liposomes is $130 \mathrm{~nm}$, we can estimate the surface charge density of the vesicles to be $\Sigma=0.33 \mathrm{C} / \mathrm{m}^{2}$. The length of spermidine molecules is $l=1.0 \mathrm{~nm}$. We treat each spermidine molecule as a rod with elementary charges located at both ends, as well as in the center of the rod.

First, we present the theoretical results obtained from the field theory. Figure 2 shows the concentration profiles of spermidine molecules (dashed thick lines) and monovalent co-ions (full thick lines). The calculations have been made for three different spermidine concentrations matching the experimental conditions: $10^{-2} \mathrm{M}, 10^{-3} \mathrm{M}$, and $10^{-4} \mathrm{M}$. Figure 2 shows increased concentration of spermidines close to the vesicle wall. The discontinuity of the first derivative of the spermidine concentration profile at around $1.5 \mathrm{~nm}$ matches the length of spermidine molecules. Far from the charged surface, the concentration of spermidines reaches their bulk value. For comparison, we also include the calculation of trivalent small mobile ions (thin lines). In the vicinity of the charged surface, the concentration of spermidines is larger than the concentration of point-like particles.

Figure 3 shows the dimensionless electrostatic potential profiles $\beta q \phi\left(z / l_{B}\right)$ in the vicinity of the surface of the charged vesicle. The full lines correspond to spermidine 
FIG. 3. Reduced electrostatic potential profiles of spermidine (full lines) and point-like ions (dashed lines) close to the charged surface: $c=10^{-4} \mathrm{M}$ (red), $c=10^{-3} \mathrm{M}$ (black), and $c=10^{-2} \mathrm{M}$ (blue).

molecules, whereas the dashed lines correspond to pointlike ions. The electrostatic potential is taken to be zero in the bulk solution. The potential is negative for rod-like. For small bulk concentrations of point-like trivalent ions the potential is negative in the vicinity of charged surface. With increasing bulk concentration (at bulk concentration of $c=10^{-4} \mathrm{M}$ (red dashed curve in Fig. 3) the potential profile switches from negative values in the vicinity of the vesicle to positive values far from the charged surface. For bulk concentrations larger than $c=10^{-3} \mathrm{M}$, the electrostatic potential remains positive.

In Fig. 4, we show the experimental electrokinetic measurements for anionic liposomes as a function of the salt concentration for $\mathrm{La}\left(\mathrm{NO}_{3}\right)_{3}$ electrolyte. As expected, a reversal in the potential is experimentally observed at a concentration of about $0.3 \mathrm{mM}$. Our theoretical model (see Fig. 4) fits fairly well with the experimental results. It should be stressed that a reversal concentration of $0.1 \mathrm{mM}$ of lanthanum was previously reported for pure PS liposomes [14].

In Fig. 5, the measurements corresponding to a spermidine electrolyte are plotted. In contrast to results of lanthanum, a reversal in the electrokinetic potential of anionic liposomes is not observed. The magnitude of the zeta-potential decrease as the spermidine concentration increases, but a mobility reversal is not conclusively observed. Moreover, for salt concentration above $10^{-2} \mathrm{M}$, liposomes tend to aggregate. It has been reported that while CR induced by spermidine is strong enough to stop the translocation of the polymer [54], this CR cannot promote a reversal in the electrophoretic mobility of DNA [36]. At this stage, it is important to clarify the concept of $\mathrm{CR}$; if the electrostatic potential of a charged surface
FIG. 4. The zeta-potential of liposomes as a function of lanthanum concentration. The red symbols corresponds to the experimental results, whereas the green symbols are the theoretical predictions.

FIG. 5. The zeta-potential of liposomes as a function of spermidine concentration. The red symbols correspond to the experimental results, whereas the green symbols are the theoretical predictions.

immersed in an electrolyte solution is calculated as function of the distance from the surface, CR can induce a reversal in the potential at distances that do not correspond with the shear plane, at which the zeta-potential is calculated [9]. Anyhow, in order to determine the reason why $\mathrm{La}^{3+}$ and spermidine, both trivalent cations, induce a dissimilar electrokinetic behavior of anionic liposomes, a rod-like ionic model is applied in Fig. 5. As can be seen, the theory matches fairly well experimental data and non-reversal in the zeta-potential is predicted.

In order to further elucidate this behavior, the concentration profiles of spermidine molecules and monovalent co-ions are plotted in Fig. 2.

Our results show that there is a substantial difference between rod-like and point-like ions. Although they have the same overall charge, the structure of the charge distribution within each particular ion influences the concentration profiles of ions [51? ]. Point-like ions can more easily come close to the charged surface. On the 
contrary, due to entropic losses near surfaces, the rodlike ions are not able to screen the charged surfaces as strongly as the point-like ions.

\section{CONCLUSIONS}

In this work, we perform new experiments examining the interaction between PS-PC liposomes in the presence of spermidine. The observed electrokinetic behavior of liposomes in spermidine is different to that found for similar liposomes in the presence of trivalent monoatomic cations, such as lanthanum. Spermidine does not induce a charge reversal in the zeta-potential. In order to explain this experimental finding, an analysis of the interaction between liposomes and spermidine is done by means of a theory that accounts for intra-ionic correlations. The theoretical approach shows important differences when the counterion is modelled as a point-like charge or when it is considered a rod-like ion. Only the latter provides a reasonable description of the experimental data. As a consequence, our main conclusion is that the distribution of the ionic charge plays an important role in the electrokinetic behavior of soft colloids in the presence of multivalent ions. Then, an appropriate theoretical framework has to be used to study the ion-colloid interaction in the case of non-monoatomic ions.

\section{ACKNOWLEDGMENTS}

KB thanks the Slovenian Research Agency for support through program P3-0388. MQP and AMM thank financial support from the Ministerio de Economía y Competitividad, Plan Estatal de Investigación Científica y Técnica y de Innovación 2013-2016 (Projects FIS201680087-C2-1-P, FIS2016-80087-C2-2-P, and FIS201681924-REDT) and the European Regional Development Fund (ERDF).
[1] J. Bentz and H. Ellens, Colloids and Surfaces 30, 65 (1988).

[2] S. Ohki and K. Arnold, Colloids and Surfaces BBiointerfaces 18, 83 (2000).

[3] T. Tanaka and M. Yamazaki, Langmuir 20, 5160 (2004).

[4] Z. D. Schultz, I. M. Pazos, F. K. McNeil-Watson, E. N. Lewis, and I. W. Levin, Journal of Physical Chemistry B 113, 9932 (2009).

[5] S. Roldán-Vargas, A. Martín-Molina, M. Quesada-Pérez, R. Barnadas-Rodríguez, J. Estelrich, and J. CallejasFernández, Physical Review E 75, 21912 (2007).

[6] S. Roldán-Vargas, R. Barnadas-Rodríguez, A. MartínMolina, M. Quesada-Pérez, J. Estelrich, and J. CallejasFernández, Physical Review E 78, 10902 (2008).

[7] J. Sabín, G. Prieto, P. V. Messina, J. M. Ruso, R. Hidalgo-Alvarez, and F. Sarmiento, Langmuir 21, 10968 (2005).

[8] G. Tresset, W. C. D. Cheong, and Y. M. Lam, Journal of Physical Chemistry B 111, 14233 (2007).

[9] J. Faraudo and A. Travesset, Journal of Physical Chemistry C 111, 987 (2007).

[10] J. Faraudo and A. Travesset, Colloids and Surfaces A: Physicochemical and Engineering Aspects 300, 287 (2007).

[11] G. Tresset, Physical Review E - Statistical, Nonlinear, and Soft Matter Physics 78, 1 (2008).

[12] C. Cametti, Chemistry and Physics of Lipids 155, 63 (2008).

[13] W. Bu, K. Flores, J. Pleasants, and D. Vaknin, Langmuir 25, 1068 (2009).

[14] A. Martín-Molina, C. Rodríguez-Beas, and J. Faraudo, Physical review letters 104, 168103 (2010).

[15] A. Martín-Molina, C. Rodríguez-Beas, and J. Faraudo, Biophysical Journal 102, 2095 (2012).

[16] Y. Levin, Reports on Progress in Physics 65, 1577 (2002).

[17] M. Quesada-Pérez, E. González-Tovar, A. MartínMolina, M. Lozada-Cassou, and R. Hidalgo-Álvarez, ChemPhysChem 4, 234 (2003).
[18] S. Pianegonda, M. C. Barbosa, and Y. Levin, Europhysics Letters (EPL) 71, 831 (2005).

[19] C. Holm (Springer Berlin Heidelberg, Berlin, Heidelberg, 2005), pp. 475-488.

[20] J. Lyklema, Colloids and Surfaces A: Physicochemical and Engineering Aspects 291, 3 (2006).

[21] A. Travesset and S. Vangaveti, Journal of Chemical Physics 131, 1 (2009).

[22] M. Quesada-Pérez, A. Martin-Molina, F. GalisteoGonzález, and R. Hidalgo-Alvarez, Molecular Physics 100, 3029 (2002).

[23] A. Martin-Molina, M. Quesada-Pérez, F. GalisteoGonzález, and R. Hidalgo-Âlvarez, Colloids and Surfaces A: Physicochemical and Engineering Aspects 222, 155 (2003).

[24] S. Ravindran and J. Wu, Langmuir 20, 7333 (2004).

[25] A. Martín-Molina, J. A. Maroto-Centeno, R. HidalgoAlvarez, and M. Quesada-Pérez, The Journal of chemical physics 125, 144906 (2006).

[26] G. I. Guerrero-García, E. González-Tovar, M. ChávezPáez, and M. Lozada-Cassou, The Journal of chemical physics 132, 54903 (2010).

[27] Z.-y. Wang and Y.-q. Ma, Phys. Rev. E 85, 62501 (2012).

[28] Z.-Y. Wang and Z. Ma, Journal of Chemical Theory and Computation 12, 2880 (2016).

[29] J. G. Ibarra-Armenta, A. Martín-Molina, K. Bohinc, and M. Quesada-Pérez, The Journal of chemical physics 137, 224701 (2012).

[30] K. Bohinc, J. M. A. Grime, and L. Lue, Soft Matter 8, 5679 (2012).

[31] J. Reščič, D. Kovačević, M. Tomšič, A. Jamnik, S. Ahualli, and K. Bohinc, Langmuir 30, 9717 (2014).

[32] V. A. Bloomfield, Current Opinion in Structural Biology 6, 334 (1996).

[33] L. C. Gosule and J. A. Schellman, Nature 259, 333 (1976).

[34] T. Thomas and T. J. Thomas, Cellular and Molecular Life Sciences CMLS 58, 244 (2001). 
[35] E. Raspaud, M. Olvera de la Cruz, J. L. Sikorav, and F. Livolant, Biophysical Journal 74, 381 (1998).

[36] K. Besteman, K. Van Eijk, and S. G. Lemay, Nat Phys 3, 641 (2007).

[37] Q. A. Acton, Advances in Spermidine Research and Application: 2012 Edition: ScholarlyBrief (ScholarlyEditions, 2012).

[38] J. N. S. R. H. Ottewill, J. Colloid Interface Sci. 26, 110 (1968).

[39] A. Safran, Statistical Thermodynamics of Surfaces, Interfaces, and Membranes (Addison-Wesley Publishing Company, Colorado, 1994).

[40] A. G. Moreira and R. R. Netz, Phys. Rev. Lett. 87, 078301 (2001).

[41] R. Kjellander, S. Marčelja, R. M. Pashley, and J. P. Quirk, J. Phys. Chem. 92, 6489 (1988).

[42] M. M. Hatlo and L. Lue, Soft Matter 5, 125 (2009).

[43] M. M. Hatlo, A. Karatrantos, and L. Lue, Phys. Rev. E 80, 061107 (2009).
[44] K. Bohinc, J. Reščič, S. Maset, and S. May, J. Chem. Phys. 134, 074111 (pages 9) (2011).

[45] S. Maset and K. Bohinc, J. Phys. A 40, 11815 (2007).

[46] V. B. Teif and K. Bohinc, Prog. Biophys. Mol. Biol. 105, 208 (2011).

[47] S. Maset, J. Reščič, S. May, J. I. Pavlič, and K. Bohinc, J. Phys. A 42, 105401 (2009).

[48] Y. W. Kim, J. Yi, and P. A. Pincus, Phys. Rev. Lett. 101, 208305 (2008).

[49] J. M. A. Grime, M. O. Khan, and K. Bohinc, Langmuir 26, 6343 (2010).

[50] M. M. Hatlo and L. Lue, EPL 89, 25002 (2010).

[51] K. Bohinc and L. Lue, Journal of nanoscience and nanotechnology 15(5), 3468 (2015).

[52] A. Rodríguez-Pulido, A. Martín-Molina, C. Rodríliqguez-Beas, O. Llorca, E. Aicart, and E. Junquera, J. Phys. Chem. B 113, 15648 (2009).

[53] K. Bohinc, J. M. A. Grime, and L. Lue, Soft Matter 8, 5679 (2012).

[54] B. S and A.-N. T, Langmuir 30, 12907 (2014). 